\title{
Sencillas maniobras del examen físico pueden adelantar el rastreo de osteoporosis o sugerir fractura vertebral
}

\section{Objetivo}

Valorar la seguridad y precisión del examen físico para el diagnóstico de osteopenia, osteoporosis (OP) o fractura vertebral.

\section{Fuentes de Datos}

Búsqueda en MEDLINE (1966-2004) ejecutada independientemente por dos revisores. Se examinaron las referencias, se consultaron 4 libros de texto de destrezas clínicas y a expertos.

\section{Selección de estudios}

Dos revisores seleccionaron independientemente estudios con datos originales adecuados sobre exactitud o precisión del examen físico para diagnosticar osteopenia, osteoporosis o fractura vertebral (test de referencia* requerido: puntaje $T-1$ a $-2.4 ; \leq-2,5$ o disminución $\geq 20 \%$ de la altura vertebral respectivamente).

\section{Extracción de datos}

Se extrajeron los datos y calificaron los estudios incluidos: Nivel 1 selección de pacientes $(=0$ más 100$)$ consecutivos y representativos, independientemente de resultados de la prueba y del test de referencia; Nivel 2 < de 100 pacientes estudiados y Nivel 3 como el nivel 2 pero con una población no consecutiva o representativa. Se excluyeron estudios de menor nivel. Se contactaron a los autores si los datos no permitían el cálculo de coeficientes de probabilidad (likelihood ratios)*.

\section{Resultados principales}

De 191 estudios identificados como relevantes 14 cumplieron los criterios de inclusión. En la Tabla figuran algunos resultados de las pruebas con mayores coeficientes de probabilidad positivos (CP+) y de mejor nivel de evidencia en el diagnóstico de Osteoporosis y de fractura vertebral (éstos últimos ilustrados en la figura). Aunque la pérdida de altura es una herramienta del examen potencialmente útil, su generalización es incierta debido a la menor calidad de los estudios que la evaluaron. La diferencia entre el largo de brazos y la altura aumenta con los años, pero al evaluar la evidencia se concluyó que no predice OP o fracturas. Tampoco son aplicables la cifosis, el grosor del pliegue cutáneo ni la fuerza prensil por inconsistencia de resultados entre estudios o malas características operativas.

Tabla. Principales signos y síntomas para el diagnóstico de osteoporosis y fractura vertebral

\begin{tabular}{l|c|c|c|c|c|c}
\hline & Valor & $\begin{array}{c}\text { Sensibi- Especifi- } \\
\text { Iidad }\end{array}$ & $\begin{array}{c}\text { Oidad } \\
\text { Osteoporosis }\end{array}$ & CP+ (IC 95\%) & CP- (IC 95\%) \\
\hline Peso & $<51 \mathrm{~kg}$ & $22 \%$ & $97 \%$ & $7,3(5,0-10,8)$ & $0,8(0,7-0,9)$ \\
& $<60 \mathrm{~kg}$ & $82 \%$ & $56 \%$ & $1,93(1,8-2,0)$ & $0,3(0,3-0,4)$ \\
\hline Nro. de dientes & 20 dientes & $27 \%$ & $92 \%$ & $3,4(1,4-8,0)$ & $0,8(0,6-1,0)$ \\
\hline Auto-reporte de espalda encorvada & - & $21 \%$ & $97 \%$ & $3,0(2,2-4,1)$ & $0,85(0,8-0,9)$ \\
\hline \multicolumn{7}{|c}{ Fractura vertebral } \\
\hline Distancia pared-occipucio & $>0 \mathrm{~cm}$ & $60 \%$ & $87 \%$ & $4.6(2.9-7.3)$ & $0.5(0.3-0.6)$ \\
\hline Distancia costilla-pelvis & $<2$ dedos & $88 \%$ & $46 \%$ & $3.8(2.9-5.1)$ & $0.6(0.5-0.7)$ \\
\hline
\end{tabular}

CP: coeficiente de probabilidad. Ver definiciones de sensibilidad, especificidad, y CP en glosario.

\section{Conclusiones}

En pacientes sin recomendación para medir la densidad mineral ósea, varias maniobras del examen físico, especialmente el bajo peso, pueden cambiar significativamente la probabilidad de osteoporosis y adelantar el rastreo. La distancia pared-occipucio $>0 \mathrm{~cm}$ y la distancia costilla-pelvis $<2$ dedos sugieren la presencia de fractura vertebral oculta.

Fuente de financiamiento: National Health Service, Reino Unido.

\section{Comentario}

La consecuencia más temida de la OP es la fractura de cadera, por la pérdida de independencia y movilidad (en muchos casos permanente) y hasta un $20 \%$ de mortalidad al año de ocurrida la misma. Además su incidencia se duplicará en los próximos 15 años de no tomarse medidas preventivas. ${ }^{1}$ La Fuerza de Tareas Estadounidense de Prácticas Preventivas (USPSTF) recomienda densitometría ósea a toda mujer con factores de riesgo de OP o mayor de 65 años. ${ }^{2}$ En mujeres más jóvenes con pocos factores de riesgo el examen físico puede contribuir a adelantarles el rastreo. También a identificar mujeres con fracturas vertebrales ocultas (2/3 de las fracturas vertebrales) presentes en más del $10 \%$ de las mujeres entre los 50 y 64 años. ${ }^{3}$ Éstas requieren tratamiento, independientemente de la densidad mineral ósea, pues aumentan 2 a 3 veces el riesgo de nuevas fracturas. ${ }^{4}$

Ninguna maniobra por sí sola es suficiente para confirmar o descartar osteoporosis o fractura vertebral sin exámenes adicionales, pero las enumeradas en la tabla modifican la probabilidad de padecerlas, especialmente si están presentes. Las reglas de predicción de OP tienen mejores $\mathrm{CP}$ - que el examen físico y pueden reducir la necesidad de densitometría en las mujeres de bajo riesgo. La validación de otras reglas en población de nuestro país no mostró tener muy buenas características operativas. ${ }^{5}$ Sin embargo una regla denominada $\mathrm{PROBA}^{6}$ (desarrollada y validada en nuestra población) que considera solo edad y peso, pudo reducir la tasa de falsos negativos al $6 \%$, y tuvo un $\mathrm{CP}+=1,57$ y $\mathrm{CP}-=0,29$ ).

\section{Conclusiones del comentador}

La presencia de sencillas maniobras del examen físico aumentan la probabilidad de OP y son útiles para adelantar el rastreo o sugerir fractura vertebral, implicando conductas terapéuticas beneficiosas de confirmarse el diagnóstico. Si las maniobras son negativas, aunque en menor medida que las reglas de predicción, también se reduce la probabilidad de OP y de estudios innecesarios.

\section{Dr. Agustín Ciapponi [ Unidad de Medicina Familiar y preventiva. Hospital Italiano de Buenos Aires. ]}

Ciapponi A. Sencillas maniobras del examen físico pueden adelantar el rastreo de osteoporosis o sugerir fractura vertebral. Evid actual pract ambul 2005;8:3940. Comentado de Green AD, Colon-Emeric CS, Bastian L, Drake MT, Lyles KW. Does this woman have osteoporosis? JAMA 2004;292:2890-2900. PMID: 15598921

\section{Referencias}

1. Riggs BL, Melton LJ 3rd.The worldwide problem of osteoporosis: insights afforded by epidemiology. Bone. 1995;17(5 Suppl):505S-511S

2. Nelson HD, Hefland M, Woolf SH, Allan JD. Screening for postmenopausal osteoporosis: a review of the evidence for the US Preventative Services Task Force. Ann Intern Med. 2002;137:529-41.

3. Melton LJ III, Lane AW, Cooper C, Eastell R, O'Fallon WM, Riggs BL. Prevalence and incidence of vertebral deformities. Osteoporos Int. 1993;3:113-9.

4. National Osteoporosis Foundation. Physician's Guide to Prevention and Treatment of Osteoporosis. Washington, DC: National Osteoporosis Foundation; 2000.

5. Ciapponi A, Mejía R, Granel A, Galich AM. Validación de la regla de predicción de Osteoporosis ORAI (Osteoporosis Risk Assesment Instrument) en mujeres de la ciudad de Buenos Aires. Presentado en el XIII Congreso Anual de Medicina Interna General. 22 y 23 de Agosto 2003

6. Ciapponi A, Mejía R, Granel A, Galich AM. Prediction Rule of Osteoporosis in Buenos Aires (PROBA), development and validation. (2004). Journal of General Internal Medicine 19 (s1), 242-260.doi: 10.1111/j.1525-1497.2004.019S1.x. Ciapponi A, 116682 p. 195 
Figura. Maniobras del examen físico para la detección fractura vertebral
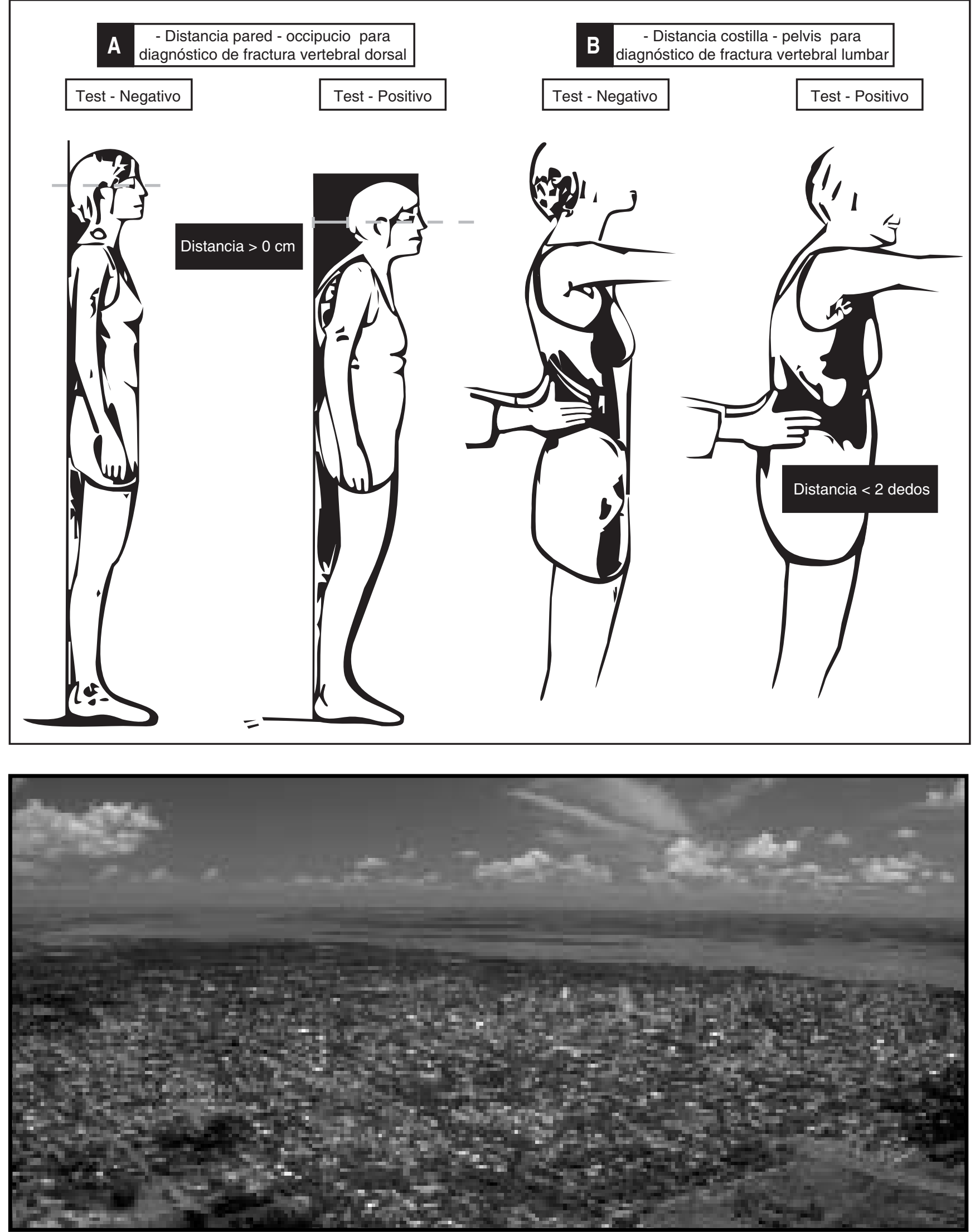

Gentileza Secretaria de Turismo de La Nación 